\title{
BMJ Open Glenoid failure after total shoulder arthroplasty with cemented all- polyethylene versus metal-backed implants: a systematic review protocol
}

\author{
Renato Aroca Aroca Zan (D) , ${ }^{1}$ Rafael Fuchs Lazarini, ${ }^{2}$ Fabio Teruo Matsunaga, ${ }^{1}$ \\ Nicola Archetti Netto, ${ }^{1}$ João Carlos Belloti, ${ }^{1,3}$ Marcel Jun Sugawara Tamaoki ${ }^{1}$
}

To cite: Zan RAA, Lazarini RF, Matsunaga FT, et al. Glenoid failure after total shoulder arthroplasty with cemented all-polyethylene versus metalbacked implants: a systematic review protocol. BMJ Open 2020;10:e043449. doi:10.1136/ bmjopen-2020-043449

- Additional material is published online only. To view, please visit the journal online (http://dx.doi.org/10.1136/ bmjopen-2020-043449)

Received 03 August 2020 Revised 24 October 2020 Accepted 02 December 2020

Check for updates

C Author(s) (or their employer(s)) 2020. Re-use permitted under CC BY-NC. No commercial re-use. See rights and permissions. Published by BMJ.

${ }^{1}$ Orthopedics and Traumatology—Division of Hand surgery and Upper Limb, Universidade Federal de São Paulo, São Paulo, São Paulo, Brazil

${ }^{2}$ Department of Orthopaedics and Traumatology, Hospital Felicio Rocho, Belo Horizonte, Minas Gerais, Brazil

${ }^{3}$ Universidade Federal de Sao Paulo Escola Paulista de Medicina, São Paulo, Brazil

Correspondence to

Dr Renato Aroca Aroca Zan; re_zan@hotmail.com

\section{ABSTRACT}

Introduction Anatomical total shoulder arthroplasty (TSA) is an effective treatment adopted for patients with glenohumeral osteoarthritis (OA). The glenoid component failure is the main risk that occurs in this therapeutic choice; however, doubts remain regarding the selection of the best implant for avoiding complication. This systematic review aims to evaluate the glenoid component in TSA by comparing the complications of different types of implants. Methods and analysis A systematic review of randomised clinical trials or quasi-randomised trials will be performed by applying the Preferred Reporting Items for Systematic Review and Meta-Analysis protocols and comparing polyethylene (keeled and pegged) versus metal-backed implants in adult patients with glenohumeral OA. Our search strategy will be performed using MEDLINE, PubMed, Cochrane Central Register of Controlled Trials, EMBASE and Web of Science. Data management and extraction will be performed using a data withdrawal form and by analysing study method characteristics, participant characteristics, intervention characteristics, results and methodological domains. The database search will be performed by February 2021. The Grading of Recommendations Assessment, Development and Evaluation will be used for assessing the quality of evidence of each study selected; however, some critical and important outcomes were determined such as the shoulder function through functional scores (ConstantMurley and American Shoulder and Elbow Surgeons), complications represented by pain (Visual Analogue Scale), surgical revision, radiograph radiolucency and loosening. The confidence in estimated effects for these outcomes will be applied as the overall confidence. The outcomes will be defined as early or late, according to the postoperative follow-up of less than or greater than 1 year, respectively, for complications and radiographs. For the shoulder function, follow-ups will be divided into 6,12 and 24 months. Heterogeneity is expected in systematic reviews; therefore, the selection of outcomes, as well as the sample size, and specific statistical analysis can lead to meta-analysis; however, if it fails, narrative evidence synthesis will be conducted. Other analyses such as descriptive, subgroup and sensitivity analyses will be performed whenever possible. This systematic review will, therefore, provide evidence concerning the best clinical practice for avoiding complications.
Strengths and limitations of this study

- This systematic review will be conducted in response to a gap in the evidence regarding an increasing number of shoulder surgical procedures performed for treating shoulder osteoarthritis $(O A)$.

- This review will include only randomised and nonrandomised controlled trials for assessing all relevant available evidence regarding the types of glenoid implants for total shoulder arthroplasties for shoulder $\mathrm{OA}$.

- A comprehensive search will be performed across several databases with no restrictions for language, date and status of publication.

- We expect difficulty in finding trials with adequate sample size, standardisation of the functional scores, follow-up pattern and methods of the results, indicating a possible limitation in our revision.

- All authors of this review have expertise in methodology in systematic reviews as well as experience in orthopaedic surgical procedures that will ensure relevance to applicability and practice.

Ethics and dissemination This study has been approved by the Institutional Review Board of Universidade Federal de São Paulo (protocols 0725/2017, 2.157.415 and 70473017.5.0000.5505), and the findings will be disseminated through peer-reviewed publication and conference presentations.

PROSPERO registration number CRD42018079537.

\section{INTRODUCTION}

Osteoarthritis (OA) of the glenohumeral joint is a common clinical condition that affects adult population between 60 and 80 years old. ${ }^{2}$ Total shoulder arthroplasty (TSA) has been proven to be effective for treating this condition. ${ }^{3}$ Utilisation of TSA increased between $300 \%$ and $400 \%$ for the last two decades (1990-2010), varying from 13000 to 42000 approximately, with an annual variation of $10.6 \%{ }^{45}$ Approximately $24 \%$ of complications of TSA were related to 
glenoid implant, and $28.5 \%$ of those required surgical revision owing to loosening of the implant. Metal-backed (MB) glenoid component's thickness is approximately 7 $\mathrm{mm}$ (4 $\mathrm{mm}$ for the polyethylene (PE) insert and $3 \mathrm{~mm}$ for the metal tray); two screws provided initial stability, and a porous back surface provided bone ingrowth ${ }^{6}$; in contrast, PE component thickness is approximately 3-4 $\mathrm{mm}^{7}$; it is fixed across the glenoid surface through pegs or keel requiring cement and its elasticity modulus is 0.5 $\mathrm{GPa}$, which is closest to cancellous $(0.4 \mathrm{GPa})$ and cortical $(2.0 \mathrm{GPa})$ bones and far from metal (cobalt/chrome (200 GPa) and titanium (112 GPa) $).{ }^{7}$ Loosening of the glenoid implant is the main cause of failure, followed by pain and decrease in the range of motion after a TSA..$^{8-11}$ This complication compromises the function of the joint and reoperation might be needed.

This systematic review aims to evaluate the glenoid component in TSA by comparing the complications of different types of implants, either with MB or PE components (keeled or pegged), considering the function of the shoulder, complications (persistence or worsening of pain and failure of the surgery with regard to the implant loosening in the glenohumeral joint leading to a revision surgery) and radiograph radiolucency.

\section{METHODS AND ANALYSIS}

\section{Types of studies and inclusion criteria}

This systematic review will follow the recommendations proposed by the Cochrane Handbook of Interventions Reviews ${ }^{12} 13$ and Preferred Reporting Items for Systematic Review and Meta-Analysis protocols. ${ }^{14} 15$ Our study will include only randomised or quasi-randomised controlled clinical trials, comparing $\mathrm{MB}$ glenoid designs and $\mathrm{PE}$ designs (keeled or pegged) for TSA; other studies such as experimental, cadaveric, cohort, observational, case report and case-control will be excluded. Small samples of $<5$ participants will not be eligible. We expect difficulty in finding trials with adequate sample size.

\section{Ethics and dissemination}

The study has been approved by the Institutional Review Board of Universidade Federal de São Paulo (protocols 0725/2017, 2.157.415 and 70473017.5.0000.5505)

Systematic review registration PROSPERO, CRD 42018079537.

\section{Types of participants (inclusion and exclusion criteria)}

Eligible articles with adults patients ( $>18$ years old) who underwent TSA, with cemented pegs or keel PE or MB, owing to idiopathic or inflammatory $\mathrm{OA}^{16-19}$ will be included in this study. The following exclusion criteria will be adopted: patients with previous surgery, neurological diseases (Charcot's arthropathy, Parkinson's disease, etc), revision surgeries of arthroplasty, reverse total arthroplasty and studies assessing other types of glenoid implants or even mixed arthroplasties (ie, use of bone graft).

\section{Primary outcomes (critical)}

Shoulder function will be assessed with 6,12 and 24 months of postoperative follow-ups, with two validated scores, Constant-Murley $(\mathrm{CM})^{20}$ and American Shoulder and Elbow Surgeons (ASES) ${ }^{21}$; the analysis is made on the following aspects: activity level, range of motion, arm positioning, usage of pain killers and work. Complications such as persistence or worsening of pain (Visual Analogue Scale (VAS) $)^{22}$ and loosening or breakage of implanted materials can lead to a surgical revision. These outcomes will be assessed as early or late, according to the postoperative follow-up of less than or greater than 1 year.

\section{Secondary outcomes (important)}

Radiolucency will be assessed by the occurrence of radiographic lines between the glenoid implant/cement and the native bone, indicating the loosening of the implant. Lazarus classification for keeled components and Franklin classification for pegged components will be used for assessing radiolucency concerning all-PE components. ${ }^{823}$ This outcome will be assessed as early or late, according to the postoperative follow-up of less than or greater than 1 year.

\section{Search methods and strategy}

The electronic search will be performed in February 2021 using MEDLINE (PubMed), Cochrane Central Register of Controlled Trials, ${ }^{24}{ }^{25}$ EMBASE, Web of Science, International Clinical Trials Registry Platform, ClinicalTrials. gov and Literatura Latino-Americana e do Caribe em Ciências da Saúde (for randomised or quasi-randomised controlled trials). The grey literature will also be searched using Google Scholar, OpenGrey and GreyNet. ${ }^{26} \mathrm{~A}$ medical librarian expert and a discussion group will conduct effective search strategy.

The following terms will be used in different combinations and combinations for our search: $((()($ "arthroplasty, replacement, shoulder" [MeSH Terms] OR ("arthroplasty" [All Fields] AND "replacement" [All Fields] AND "shoulder"[All Fields]) OR "shoulder replacement arthroplasty"[All Fields] OR ("total"[All Fields] AND "shoulder"[All Fields] AND "arthroplasty"[All Fields]) OR "total shoulder arthroplasty"[All Fields]) AND glenoid[All Fields]) AND loosening[All Fields]) OR keeled[All Fields]) OR pegged[All Fields]) OR metalbacked[All Fields]) AND radiolucency[All Fields]. There will be no restriction on language or publication status. Full search strategies for the main databases are provided in online supplemental appendix 1.

\section{Data collection and analysis}

Two independent reviewers will access the selected studies and the extracted data from these studies using EndNote V.X9 (Clarivate Analytics, Boston, Massachusetts, USA), to facilitate collaboration among them during the selection process.

Two authors will independently select and analyse the eligible studies for this systematic review through the title 
and abstract using the following criteria: (1) randomised clinical trials or quasi-randomised trials, (2) TSA with cemented glenoid PE or MB and (3) TSA loosening after $\mathrm{PE}$ or MB. Selected studies will be entirely reviewed for determining their eligibility, and any disagreement will be solved through discussion and, when necessary, will be judged by a third author in an attempt to resolve a possible conflict.

Based on the population, intervention, comparisons and outcomes, ${ }^{27} 28$ the results will be established for each outcome, the magnitude of the effects and the assessment of the quality of evidence $(\mathrm{QE})$, besides the five reasons (risk of bias, imprecision, inconsistency, indirectness and risk of publication bias) that can lower the confidence in those estimated effects, downgrading the QE.

\section{Data extraction and handling}

Data extraction will be performed by two reviewers; data will be extracted using an appropriate customised extraction form (Microsoft Access/Excel, Excel V.16.34. 2020), based on (1) methodological characteristics, including design and duration, whether the protocol was published prior to the recruitment of the patients, possible funding sources and study registration; (2) characteristics of the participants including location, number of recruits, their evaluation, inclusion and exclusion criteria, age and classification relevant to the disease addressed; (3) characteristics of the intervention such as duration, surgery type and complications; (4) results through time and loss of follow-up and (5) methodological domains and risk of bias. ${ }^{28} 29$

The extracted data will be further classified according to the time of follow-up as early and late, establishing 1 year as the cut-off for this division.

\section{Assessment of risk of bias}

Two authors will independently evaluate various aspects of the methodological quality of the included studies using The Grading of Recommendations Assessment, Development and Evaluation (GRADE) (www.gradepro. org $)^{30}$ for assessing limitations in study design and execution, similar to a modified version of the Cochrane Bone Joint and Muscle Trauma Group tool form. ${ }^{31}$ Some items will be considered: random sequence generation, allocation concealment, participant blinding, intention-totreat analysis properly applied, loss of follow-up, outcome assessment blinding, quality criteria such as trials that stopped early for benefit and when there are cross-over designs, selective reporting and potential influence of incomplete outcome data for each trial, will also be performed. After judgement and classification, the $\mathrm{QE}$ for each outcome will generate three levels of risk of bias: high, uncertain and low, and it can be rated by the GRADE approach depending on the 'seriousness' of bias. ${ }^{32}{ }^{33}$ Disagreements will be solved by the analysis of a third reviewer after further analysis. ${ }^{30} 31$

\section{Measures of treatment effect}

The resulting dichotomous data will be analysed with a relative risk and 95\% CI. When appropriate, the estimated effects will be expressed as numbers that need treatment measuring the complications of the two types of glenoid implants in the population of TSA. Data on continuous outcomes will be expressed as an average difference of $95 \%$ CI. The results will be grouped with the mean difference (MD) if two or more trials reveal results from the same valid instrument of evolution (with the same units of measurement). If primary studies measure the same outcomes such as shoulder function through validated scores, complications or radiograph using different instruments (as well as different units of measurement), OR will be transformed into standard MD (SMD) and effect size. The Cochrane Review Manager (computer program, V.5.3, Copenhagen: the Nordic Cochrane Centre, the Cochrane Collaboration, 2014) will be used for statistical analyses, combining SMD using inverse variance method. Selective publication of studies can lead to a false estimated effect known as 'file-drawer problem'. Small numbers of patients and studies funded by industry are also factors that negatively influence publication bias, which can be evaluated using funnel plots; less publications bias was detected when studies were distributed around the best estimate of effect (HR) ${ }^{33-36}$

\section{Missing data}

An intention-to-treat analysis will be performed to include all randomised participants of any intervention. Authors of the selected trials will be contacted regarding insufficient information according to the estimated effects as well as the number of participants, uncertainty in measurements (SD or SE) or number of events. An analysis will be performed independently of the lost data according to the worst-case and best-case scenarios. ${ }^{34}$

\section{Descriptive analysis}

All studies will be described in detail with a valid tool because of heterogeneous information, varied objectives, inclusion criteria, data collection methods, as well as participants' demographic characteristics, and each outcome.

\section{Subgroup analysis and heterogeneity investigation and analysis}

Subgroups will be analysed to explore the difference in the side effect related to the type of glenoid implant selected. ${ }^{34}$ The heterogeneity of estimated effects between the included studies will be evaluated using the following topics:

1. Split subgroups for allowing comparisons $(\mathrm{PE} \times \mathrm{MB}$, keel PExpeg PE) if trials are similar.

2. Separate factors that introduce heterogeneity using summary plot.

3. Determine relative effects.

4. Visual inspection using Florestal plot and statistical Higgins $\mathrm{I}^{2}$ test (significant $>50 \%$ ). 


\section{Data synthesis}

The results of comparative tests will be grouped using the random-effect model and a 95\% CI because of different true estimated effects between the selected studies, diversity in population or methodological characteristics. Despite study similarities, studies cannot be assumed to be identical. However, the variable model will be used when there is a diversity in clinical or methodological characteristics.

\section{Sensitivity analysis}

The effects of concealment allocation, studies at risk of bias, missing data, time bias, subpopulations, different prediagnoses and other kind of implants or surgical techniques will be investigated. Such articles will be excluded so that the quality of our primary analysis is not compromised. ${ }^{34}$

\section{Confidence in cumulative evidence}

GRADE (www.gradepro.org) will be applied to describe and rate the $\mathrm{QE}$ and the strength of recommendations, classifying them as high, moderate, low and very low ${ }^{37-39}$ according to the study design, ranging from the randomised trials (high $\mathrm{QE}$ ) to observational studies (low QE). The five categories mentioned before (risk of bias, inconsistency, indirectness, imprecision and publication bias) can lower the GRADE approach; however, large effects, dose-response relationship and all plausible residual confounders or biases (would reduce a demonstrated effect or suggest a spurious effect if no effect was observed) can upgrade the QE. ${ }^{33}$

Some critical and important outcomes for the GRADE approach were determined: shoulder function through functional scores (CM and ASES), complications represented by pain (VAS), surgical revision, radiograph radiolucency and loosening. ${ }^{40}$ These outcomes will be assessed individually, and individual recommendation will be provided.

Following this protocol publication, electronic search will be performed and the selected trials will be analysed. Once we get the results, we intend to publish this manuscript. Our intention is to have the manuscript ready by the end of 2021. We expect to observe an increasing rate of TSA in the adult population; therefore, complications also assume an increasingly important role in this particular treatment. The glenoid component is the main site of these complications in terms of pain, limiting the range of motion and worsening the quality of life. These findings are correlated with loosening or even implant breakage. ${ }^{41}$ There is some evidences that cemented all-PE glenoid implant has a better loosening rate than the MB design, but in terms of radiolucency, this statement is reversed. ${ }^{642-44}$

Currently, there are several types of glenoid implants in both $\mathrm{PE}$ and $\mathrm{MB}$ designs; however, there is a lack of systematic reviews based on a literature search. Particularly, only one study was found, including trials with a low level of evidence such as non-randomised and case series. ${ }^{45}$ Further evaluation on this subject with better methodological quality should be performed for covering functional, clinical and radiographic outcomes as well as complications.

We expect difficulty in finding trials with adequate sample size, standardisation of the functional scores, follow-up pattern and methods of the results, indicating a possible limitation in our revision. Our study will serve as a guide for future trials with better methodological quality.

\section{Twitter João Carlos Belloti @jcbelloti}

Contributors All authors participated in all stages of preparation of this study; however, each one was responsible for a step in its confection. RAAZ is the guarantor of the review and drafted the manuscript. RAAZ, FTM, JCB and MJST conceptualised the methods. RAAZ and RFL contributed to the development of the eligibility criteria and data extraction items. RAAZ, FTM and MJST designed the work. NAN helped with the electronic search and translation. All authors reviewed several drafts of the manuscript for critical content and approved the final protocol.

Funding The authors have not declared a specific grant for this research from any funding agency in the public, commercial or not-for-profit sectors.

Competing interests None declared.

Patient consent for publication Not required.

Provenance and peer review Not commissioned; externally peer reviewed.

Supplemental material This content has been supplied by the author(s). It has not been vetted by BMJ Publishing Group Limited (BMJ) and may not have been peer-reviewed. Any opinions or recommendations discussed are solely those of the author(s) and are not endorsed by BMJ. BMJ disclaims all liability and responsibility arising from any reliance placed on the content. Where the content includes any translated material, BMJ does not warrant the accuracy and reliability of the translations (including but not limited to local regulations, clinical guidelines, terminology, drug names and drug dosages), and is not responsible for any error and/or omissions arising from translation and adaptation or otherwise.

Open access This is an open access article distributed in accordance with the Creative Commons Attribution Non Commercial (CC BY-NC 4.0) license, which permits others to distribute, remix, adapt, build upon this work non-commercially, and license their derivative works on different terms, provided the original work is properly cited, appropriate credit is given, any changes made indicated, and the use is non-commercial. See: http://creativecommons.org/licenses/by-nc/4.0/.

ORCID iD

Renato Aroca Aroca Zan http://orcid.org/0000-0001-9289-7983

\section{REFERENCES}

1 Neer CS, Watson KC, Stanton FJ. Recent experience in total shoulder replacement. J Bone Joint Surg Am 1982;64:319-37.

2 Rasmussen JV, Brorson S, Hallan G, et al. Is it feasible to merge data from national shoulder registries? a new collaboration within the Nordic arthroplasty register association. J Shoulder Elbow Surg 2016;25:e369-77.

3 Matsen FA. Early effectiveness of shoulder arthroplasty for patients who have primary glenohumeral degenerative joint disease. $J$ Bone Joint Surg Am 1996;78:260-4.

4 Khatib O, Onyekwelu I, Yu S, et al. Shoulder arthroplasty in New York state, 1991 to 2010: changing patterns of utilization. J Shoulder Elbow Surg 2015;24:e286-91.

5 Day JS, Lau E, Ong KL, et al. Prevalence and projections of total shoulder and elbow arthroplasty in the United States to 2015. J Shoulder Elbow Surg 2010;19:1115-20.

6 Boileau P, Moineau G, Morin-Salvo N, et al. Metal-backed glenoid implant with polyethylene insert is not a viable long-term therapeutic option. J Shoulder Elbow Surg 2015;24:1534-43.

7 Matsen FA, Lippitt SB, Rockwood CA. Chapter 16 Glenohumeral arthritis and its management. In: Rockwood CA, Matsen FA, Wirth MA, eds. Rockwood and Matsen's The Shoulder. 5th edN. Philadelphia, PA: Saunders/Elsevier, 2016: 831-1042. 
8 Franklin JL, Barrett WP, Jackins SE, et al. Glenoid loosening in total shoulder arthroplasty. association with rotator cuff deficiency. $J$ Arthroplasty 1988;3:39-46.

9 Sperling JW, Cofield RH, Rowland CM. Neer hemiarthroplasty and Neer total shoulder arthroplasty in patients fifty years old or less. long-term results. J Bone Joint Surg Am 1998;80:464-73.

10 Torchia ME, Cofield RH, Settergren CR. Total shoulder arthroplasty with the Neer prosthesis: long-term results. J Shoulder Elbow Surg 1997;6:495-505.

11 Wirth MA, Rockwood CA. Complications of total shoulderreplacement arthroplasty. J Bone Joint Surg Am 1996;78:603-16.

12 Higgins J, Green S. Cochrane Handbook for systematic reviews of interventions. version 5. London: Cochrane Collaboration, 2011.

13 McKenzie JE, Brennan SE, Ryan RE, et al. Chapter 3: Defining the criteria for including studies and how they will be grouped for the synthesis. In: Higgins JPT, Thomas J, Chandler J, et al, eds. Cochrane Handbook for systematic reviews of interventions version 6.0 (updated July 2019. Cochrane, 2019.

14 Moher D, Shamseer L, Clarke M, et al. Preferred reporting items for systematic review and meta-analysis protocols (PRISMA-P) 2015 statement. Syst Rev 2015;4:1.

15 Shamseer L, Moher D, Clarke M, et al. Preferred reporting items for systematic review and meta-analysis protocols (PRISMA-P) 2015: elaboration and explanation. BMJ 2015;349:g7647.

16 Edwards TB, Labriola JE, Stanley RJ, et al. Radiographic comparison of pegged and keeled glenoid components using modern cementing techniques: a prospective randomized study. J Shoulder Elbow Surg 2010;19:251-7.

17 Kilian CM, Press CM, Smith KM, et al. Radiographic and clinical comparison of pegged and keeled glenoid components using modern cementing techniques: midterm results of a prospective randomized study. J Shoulder Elbow Surg 2017;26:2078-85.

18 Gartsman GM, Elkousy HA, Warnock KM, et al. Radiographic comparison of pegged and keeled glenoid components. J Shoulder Elbow Surg 2005;14:252-7.

19 Rahme H, Mattsson P, Wikblad L, et al. Stability of cemented in-line pegged glenoid compared with keeled glenoid components in total shoulder arthroplasty. J Bone Joint Surg Am 2009;91:1965-72.

20 Constant $\mathrm{CR}$, Murley AH. A clinical method of functional assessment of the shoulder. Clin Orthop Relat Res 1987;214:160???164-4.

21 Michener LA, McClure PW, Sennett BJ. American shoulder and elbow surgeons standardized shoulder assessment form, patient self-report section: reliability, validity, and responsiveness. J Shoulder Elbow Surg 2002;11:587-94.

22 McCormack HM, de L. Horne DJ, Sheather S. Clinical applications of visual analogue scales: a critical review. Psychol Med 1988:18:1007-19.

23 Lazarus MD, Jensen KL, Southworth C, et al. The radiographic evaluation of keeled and pegged glenoid component insertion. $J$ Bone Joint Surg Am 2002;84:1174-82.

24 Searching for studies (August 2020): the Cochrane collaboration, 2011. Available: http://training.cochrane.org/resource/searchingstudies

25 Higgins SG. Cochrane Handbook for Systematic Reviews of Interventions Version 5.1.0 [updated Mach 2011] The Cochrane Collaboration 2011.

26 Mahood Q, Van Eerd D, Irvin E. Searching for grey literature for systematic reviews: challenges and benefits. Res. Syn. Meth. 2014;5:221-34.

27 Schardt C, Adams MB, Owens T, et al. Utilization of the PICO framework to improve searching PubMed for clinical questions. BMC Med Inform Decis Mak 2007;7:16.
28 Li T, Higgins JPT, Deeks JJ. Chapter 5: Collecting data. In: Higgins JPT, Thomas J, Chandler J, eds. Cochrane Handbook for systematic reviews of interventions version 6.1 (updated September 2020. Cochrane, 2020

29 Munn Z, Tufanaru C, Aromataris E. JBI's systematic reviews: data extraction and synthesis. Am J Nurs 2014;114:49-54.

30 Higgins JPT, Savović J, Page MJ, et al. Chapter 8: Assessing risk of bias in a randomized trial. In: Higgins JPT, Thomas J, Chandler $\mathrm{J}$, et al, eds. Cochrane Handbook for Systematic Reviews of Interventions version 6.00 [updated July 2019. Cochrane, 2019.

31 Assessing risk of bias in included studies (August 2020): the Cochrane collaboration, 2011. Available: http://methods.cochrane. $\mathrm{org} / \mathrm{bias} /$ assessing-risk-bias-included-studies

32 Guyatt GH, Oxman AD, Vist G, et al. GRADE guidelines: 4. Rating the quality of evidence--study limitations (risk of bias). J Clin Epidemiol 2011;64:407-15.

33 McKenzie JE, Brennan SE. Chapter 12: Synthesizing and presenting findings using other methods. In: Higgins JPT, Thomas J, Chandler $\mathrm{J}$, eds. Cochrane Handbook for systematic reviews of interventions version 6.1 (updated September 2020. Cochrane, 2020.

34 Deeks JJ, Higgins JPT, Altman DG. Chapter 10: Analysing data and undertaking meta-analyses. In: Higgins JPT, Thomas J, Chandler $\mathrm{J}$, eds. Cochrane Handbook for systematic reviews of interventions version 6.1 (updated September 2020. Cochrane, 2020.

35 Guyatt GH, Thorlund K, Oxman AD, et al. Grade guidelines: 13 . preparing summary of findings tables and evidence profilescontinuous outcomes. J Clin Epidemiol 2013;66:173-83.

36 Boutron I, Page MJ, Higgins JPT, et al. Chapter 7: Considering bias and conflicts of interest among the included studies. In: Higgins JPT, Thomas J, Chandler J, et al, eds. Cochrane Handbook for systematic reviews of interventions version 6.0 (updated July 2019. Cochrane, 2019.

37 Balshem H, Helfand M, Schünemann HJ, et al. Grade guidelines: 3. rating the quality of evidence. J Clin Epidemiol 2011;64:401-6.

38 Guyatt G, Oxman AD, Akl EA, et al. Grade guidelines: 1. Introduction-GRADE evidence profiles and summary of findings tables. J Clin Epidemiol 2011;64:383-94.

39 Schünemann HJ, Higgins JPT, Vist GE, et al. Chapter 14: Completing 'Summary of findings' tables and grading the certainty of the evidence. In: Higgins JPT, Thomas J, Chandler J, et al, eds. Cochrane Handbook for systematic reviews of interventions version 6.0 (updated July 2019. Cochrane, 2019.

40 Guyatt G, Oxman AD, Sultan S, et al. Grade guidelines: 11. making an overall rating of confidence in effect estimates for a single outcome and for all outcomes. J Clin Epidemiol 2013;66:151-7.

41 Boileau P, Avidor C, Krishnan SG, et al. Cemented polyethylene versus uncemented metal-backed glenoid components in total shoulder arthroplasty: a prospective, double-blind, randomized study. J Shoulder Elbow Surg 2002;11:351-9.

42 Fox TJ, Cil A, Sperling JW, et al. Survival of the glenoid component in shoulder arthroplasty. J Shoulder Elbow Surg 2009;18:859-63.

43 Walch G, Young AA, Boileau P, et al. Patterns of loosening of polyethylene keeled glenoid components after shoulder arthroplasty for primary osteoarthritis: results of a multicenter study with more than five years of follow-up. J Bone Joint Surg Am 2012;94:145-50.

44 Throckmorton TW, Zarkadas PC, Sperling JW, et al. Pegged versus keeled glenoid components in total shoulder arthroplasty. J Shoulder Elbow Surg 2010;19:726-33.

45 Papadonikolakis A, Matsen FA. Metal-Backed Glenoid components have a higher rate of failure and fail by different modes in comparison with All-Polyethylene components: a systematic review. J Bone Joint Surg Am 2014;96:1041-7. 